\title{
Development of a training system for interventional radiology
}

\author{
M. Ide ${ }^{1}$, Y. Fujii ${ }^{2}$, B. Fujioka ${ }^{2}$, T. Komeda ${ }^{2}$, H. Koyama ${ }^{2}$, \\ S. Yamamoto ${ }^{2}$, M. Mohri ${ }^{3}$ \& P. Beomonte Zobel ${ }^{4}$ \\ ${ }^{1}$ Shibaura Institute of Technology, Functional Control Systems, Japan \\ ${ }^{2}$ Shibaura Institute of Technology, Systems Engineering, Japan \\ ${ }^{3}$ Mohri Hospital, Japan \\ ${ }^{4}$ University of L'Aquila, facolta di ingegneria, \\ Laboratorio di Automazione a Fluido, Italy
}

\begin{abstract}
The objective of the study reported here was to develop a master slave system for catheter-guided vascular surgery conducted by interventional radiology. By using a master slave system, the surgeon is not exposed to x-rays during the operation because the master tool managed by an operator is located away from the slave tool, which is near the patient. The system must provide vivid realism to the surgeon, particularly with regard to force information, because this surgery is performed in three dimensions while the surgeon watches a two-dimensional monitor. In this study, we developed a training system for a catheter guide in order to upgrade the surgeon's skills because it is difficult to upgrade a master slave system without training. The system consists of a human interface device as the master tool, a control box, and a simulator. This training simulator is for the master slave system, which we developed. The master tool has a force display function using an electrorheological fluid. Two advantages of the fluid actuator are that it can be used without force feedback control and there is mechanical safety, as the surgeon does not experience any accidental force. An open loop control is used to achieve a simple mechanism and algorithm. Our results of preliminary experiments indicated that the output force achieved correlated with that sent from the PC. Three surgeons evaluated this training system under a variety of conditions. The operation of the master tool is simple. The thrust and rotation movements of the catheter can be handled instinctively and without complicated instructions. In addition, accurate force display, response, and stability were achieved with the electrorheological fluid. In the future, the training will need for a realistic depiction of interventional radiology, and the system provides accurate readings for aspiration and blood flow.
\end{abstract}

Keywords: interventional radiology, training system, force feedback. 


\section{Introduction}

Interventional Radiology (IR) is a minimally invasive surgery. Its use has increased in the last 20 years. IR requires a catheter approach through a blood vessel to the diseased area where the surgery is to be performed with the use of small tools. With this procedure, Digital Subtraction Angiography (DSA) is used to determine the position of the catheter within the body with a vascular contrast medium. DSA transmits a continuous $\mathrm{X}$-ray that is used to determine the position of the catheter, and, as a result, medical personnel are exposed to $\mathrm{x}$-rays even when protection is worn.

The motivation to conduct this study was the need to develop a Master Slave System (MSS) for catheter-guided IR surgery through the blood vasculature. The MSS is required to prevent the exposure of medical personnel to X-rays. The operator manages a master tool at a remote location from the slave tool, which is placed near the patient and under the DSA device.

The force display between the master and slave is important to warn the surgeons when a surgery error occurs. However, the force display is not only for patient safety. A surgeon can view the vasculature from within with the use of a monitor during the IR procedure; however, the picture is two-dimensional information, making it important to display the force in order to accurately estimate the image depth. In addition, the force display is used for error recovery as well as an assessment of the heartbeat, aspiration, and affected area. These innovations are used to achieve a higher degree of realism.

We have developed an MSS for interventional radiology [1]. However, generally, an MSS is difficult to adopt unless training is provided. Specialized training is necessary before this system can be adopted. Training to use the system is a problem due to its cost. A training system has been developed with virtual reality for cost reduction [2]. Research for the training system has indicated that training for catheterization effectively enhances the skill of the surgeon [3]. Therefore, for the purposes of this study, we developed a catheter simulator to use for teaching an advanced technique for catheterization.

The operation of catheter is possible to conduct the catheter movement in the twist and insertion direction [4]. During an IR procedure, the operator estimates the depth of the blood vessel while watching a monitor with two degrees of freedom. In this case, the force reflection is important to confirm the contact between the catheter and a blood vessel [5]. The force reflection is an advantage to the surgeon [6]. Therefore, the display of force assists the individual who places the catheter in the field of the blood vessel in three dimensions while viewing the monitor in two dimensions. As a result, patient safety is improved.

There are some previous studies involving catheter training systems [7,8]. Most of these studies focus on catheter function and movement. However, none of the existing devices displays the force reflection or heartbeat [9], and there are no specific interfaces to be used with force feedback [10] or to demonstrate differences in the actual IR image [11]. 
Recently, Mentice, Inc. (www.mentice.com) developed a Procedicus Vascular Intervention System Trainer (VIST ${ }^{\mathrm{TM}}$ ). This system, as a treatment tool, displays force in three dimensions and accurately depicts a patient's ailment. The simulator, however, does not provide an accurate display of the heartbeat, blood flow, and aspiration.

For this study, the training system was developed for an existing MSS [1]. Furthermore, although the device requires specific instruments, such as a computer board, it is generally assumed that it can be used in conjunction with a common PC connected with a universal serial bus (USB). In addition, with the use of an IR, it should be possible to assess the heartbeat, blood flow, and aspiration.

\section{Training simulator}

A system overview is shown in Fig. 1. The configuration diagram of the haptic device, console box, and simulator is shown in Fig. 2. The haptic device is used to accurately recreate an IR procedure with two degrees of freedom for insertion and rotation. The catheter with a two-dimensional image is guided toward the target by the operator. The operators were able to sense heartbeat during the training sessions.

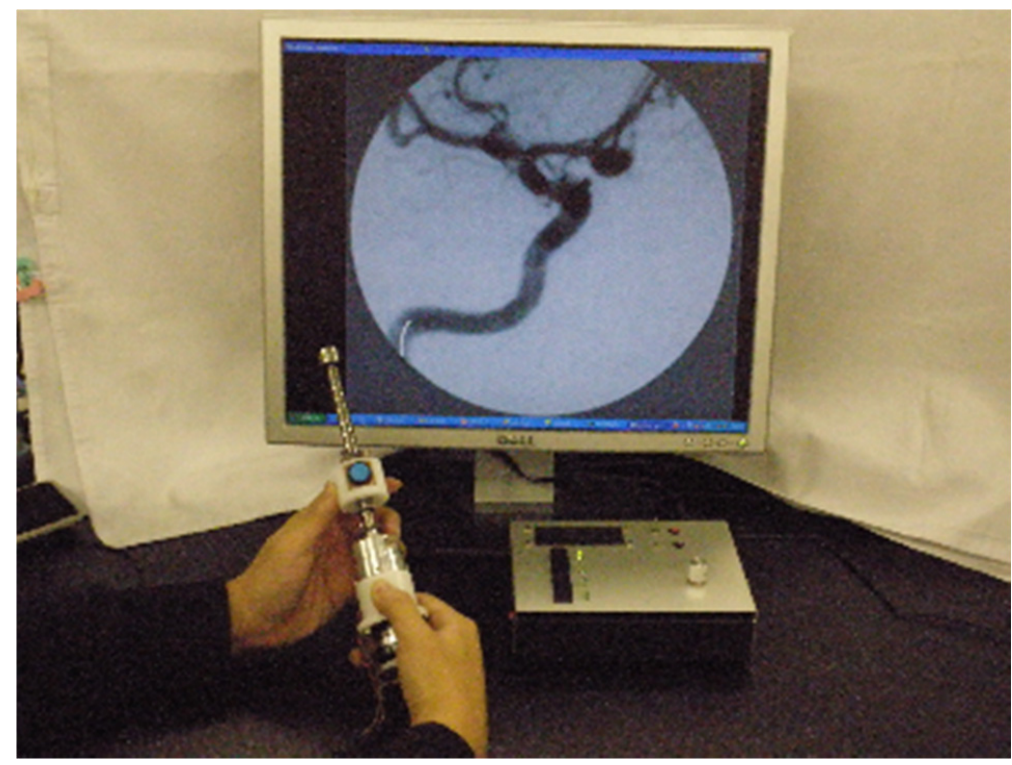

Figure 1: Overview of the training simulator. 


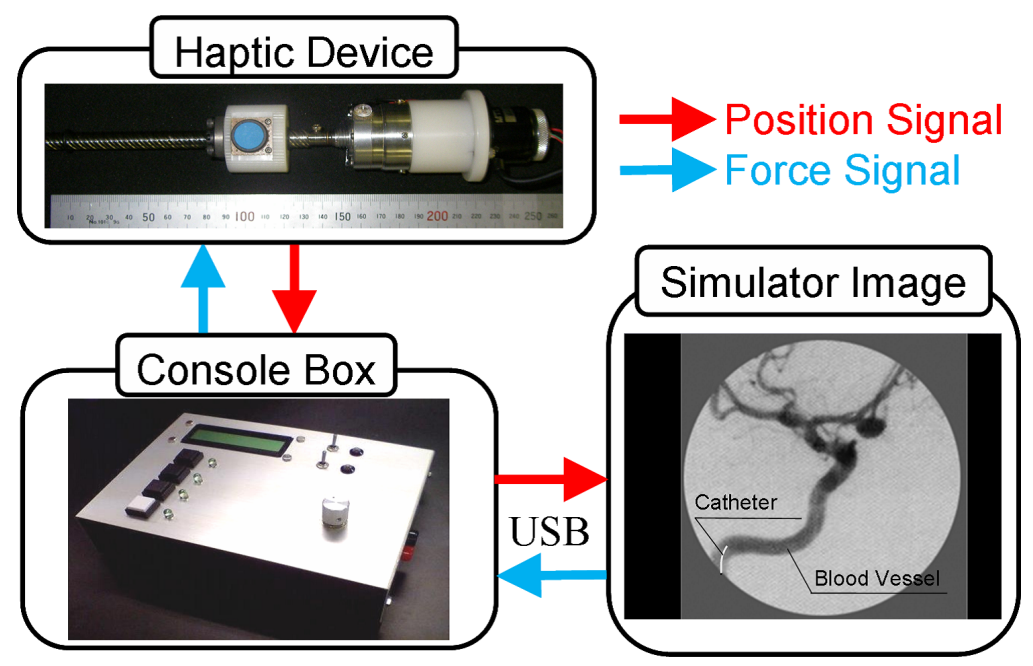

Figure 2: Diagram of the configuration of the haptic device, console box, and simulator image.

\subsection{Haptic device [1]}

This haptic device, shown in Fig. 3, has a ball screw; it rotates in synchronicity with all components and identifies the position of the thrust movement with the use of an encoder. The electrorheological (ER) fluid is placed in a case with a disk that is connected to a ball screw. The disk rotates with the ball screw, and the operator senses the force because of the resistance from the rotation caused by the voltage given to the ER fluid. The ER fluid is functional. It changes the shear viscosity or dynamic viscous elasticity according to the supplied electric field. This is the principle generating the force feedback.

The ball screw is supported by bearings, and there are two seals used to retain the ER fluid within the case. When using it, the operator grasps the outer frame and moves the nut of the ball screw forward and backward as the thrust movement. At this moment, a disk rotates within the case. If the ER fluid changes viscosity, the disk reacts to the friction created. The fluid prevents smooth movement, and the operator senses force and torque from the ball screw. In comparison to an electric motor, the manner of force display is simpler in this system. The operator receives force feedback when the thrust shafts rotate with the disk. However, the contact points on the ball screw are the ball bearings and the seals so that the friction is at a minimum for the performance of the force feedback. The sensor for position is equipped with two encoders to detect the thrust $(360[\mathrm{ppr}])$ and rotation $(360[\mathrm{ppr}])$ direction.

The operator holds the nut of the ball screw with one hand and the outer frame with the other. The operator may conduct two movements. The axis of ball screw is directly connected to the encoder axis and to the disk for the force display. The 


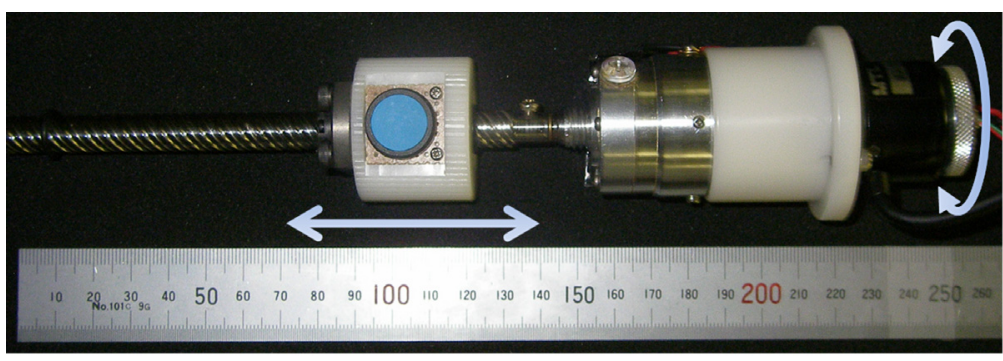

Figure 3: The haptic device has a ball screw that can rotate in synchronicity with all components and detect the position of the thrust movement using an encoder. The ER fluid is in a case with a disk that is connected to a ball screw. The disk rotates with the ball screw, and the operator can feel the force because of the resistance from the rotation caused by the voltage given to the ER fluid.

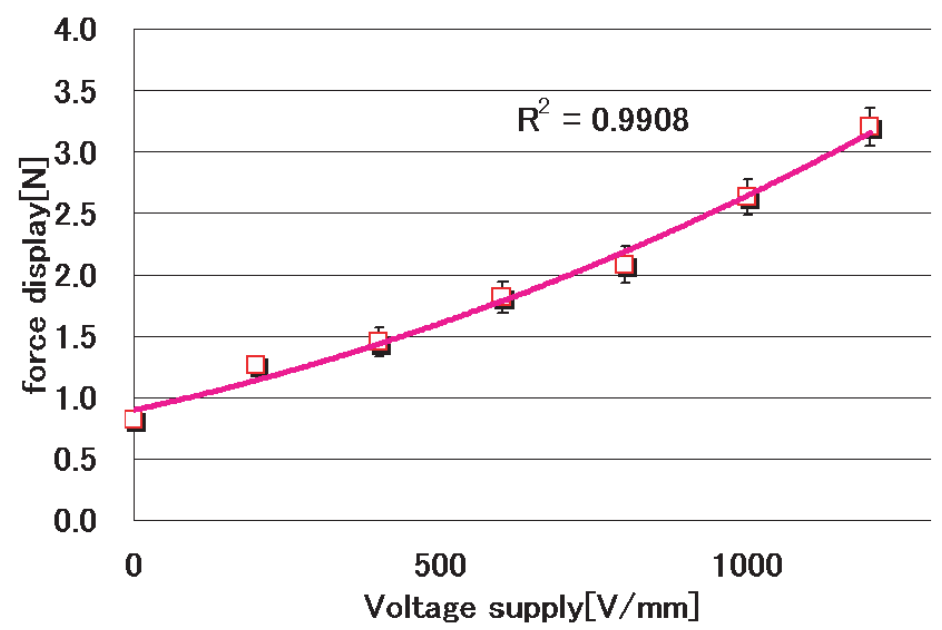

Figure 4: Volt-force curve of the haptic device.

weight of this device is about 200 [g], the length is 250 [mm], and the diameter is 48 [mm]. The volt-force curve is shown in Fig. 4.

\subsection{Console box}

The console box distributes the commands from the haptic device and the simulator. The catheter on the simulator moves on the basis of the information received from the console box. Moreover, the force on the catheter in the simulator is defined in the program and is then sent to the console box so that the collision 
force between catheter and the wall of the vessel and interference can be displayed to the operator.

Generally, controls or measurements from a PC, such as those obtained from ER fluid, an encoder, or haptic devices, require specific instruments, such as a pulse counter or DA boards. However, the simulator considered here was intended for a general case because it is difficult to use a common PC due to its lack of the required instruments. This system can be connected to a PC with a USB port.

\section{Modeling of IR}

\subsection{Modeling of catheter and wall of vessel}

A dynamic model for simulation is shown in Fig. 5. The blood vessel assumes a rigid body. When the catheter collides, the wall becomes deformed in the direction of insertion. The tip of the catheter is bent in order to determine the direction for the branch connection of the vessel. Furthermore, the catheter can also be deformed when it collides with a vessel. For realizing this condition, the catheter constructed discrete model that is connected contact point of rigid stick.

\subsection{Collision force and simulator image}

The collision force generally consists of elasticity and viscosity. However, this haptic device uses ER fluid for force reflection so that only viscosity was expressed. Only if viscosity reflection causes, the operator can sense the collision so that the patient's security can preserve because it is a passive force reflection. The image used is an angiographic picture [12]. The image was displayed on the simulation monitor with a texture mapping method of OpenGL.

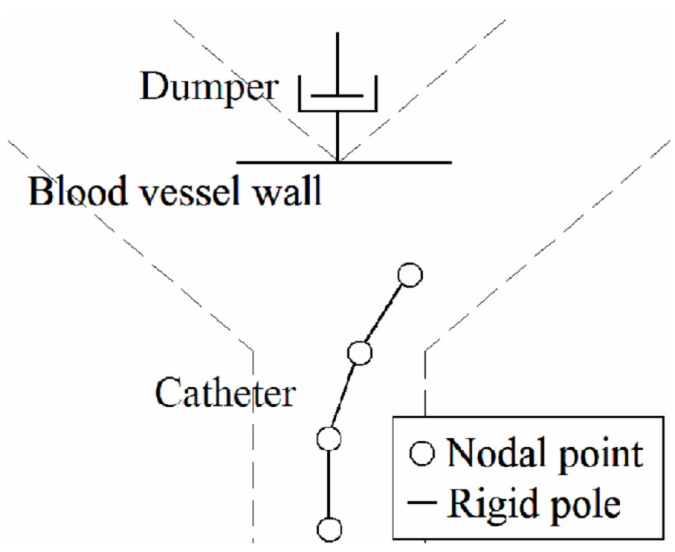

Figure 5: Dynamic simulation model. 


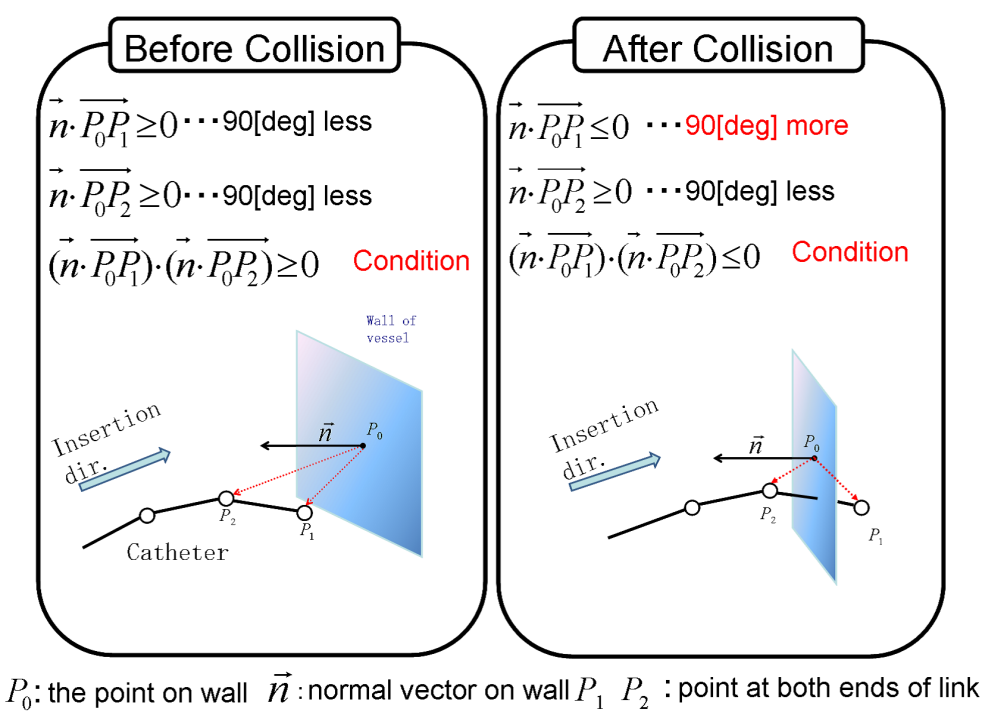

Figure 6: Principle of collision detection.

\subsection{Collision detection}

The coordinates of the parts of the wall of the vessel face on the image were extracted for collision detection. One of the links of catheter was assumed to be a segment of a line. The wall of the vessel was assumed to be a flat surface. The image of collision detection is shown in Fig. 6. The collision is detected when one joint point put front side and when other joint point put behind side as condition of collision. The joint is indicated by a point, $P_{0}$, on the vessel wall; the normal vector of the vessel wall is $\mathrm{n}$; and the points at both ends are $P_{1}, P_{2}$. The collision detection is circulated from the inner product of $P_{0} P_{1}, P_{0} P_{2}$, and vector $\mathrm{n}$. The condition equation is shown as Eq. (1).

When a collision occurs, the angle of $P_{0} P_{1}$ and vector $\mathrm{n}$ will be blunt. The value of the inner product is negative. On the other hand, the angle of $P_{0} P_{2}$ and vector $\mathrm{n}$ will be sharp so that the value of the inner product is positive. When this value is negative, a collision is detected; when it is positive, no collision is detected.

$$
\vec{n} \cdot \overrightarrow{P_{0} P_{1}} \times \vec{n} \cdot \overrightarrow{P_{0} P_{2}} \leq 0
$$

\subsection{Catheter inflection}

Although a catheter is generally made of a flexible material, such as nylon or polyurethane, in this study, the catheter model is a polyarticular link mechanism capable of inflection for simplification. As shown in Fig. 7, the operator inserts the catheter in the direction of insertion, and the simulator detects the collision. 

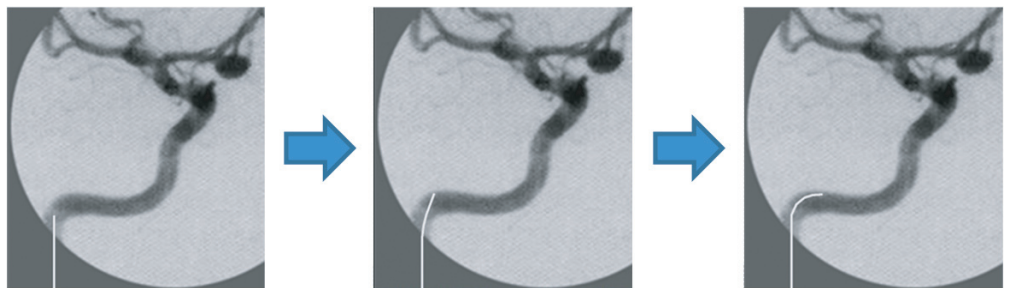

Figure 7: Catheter inflection.

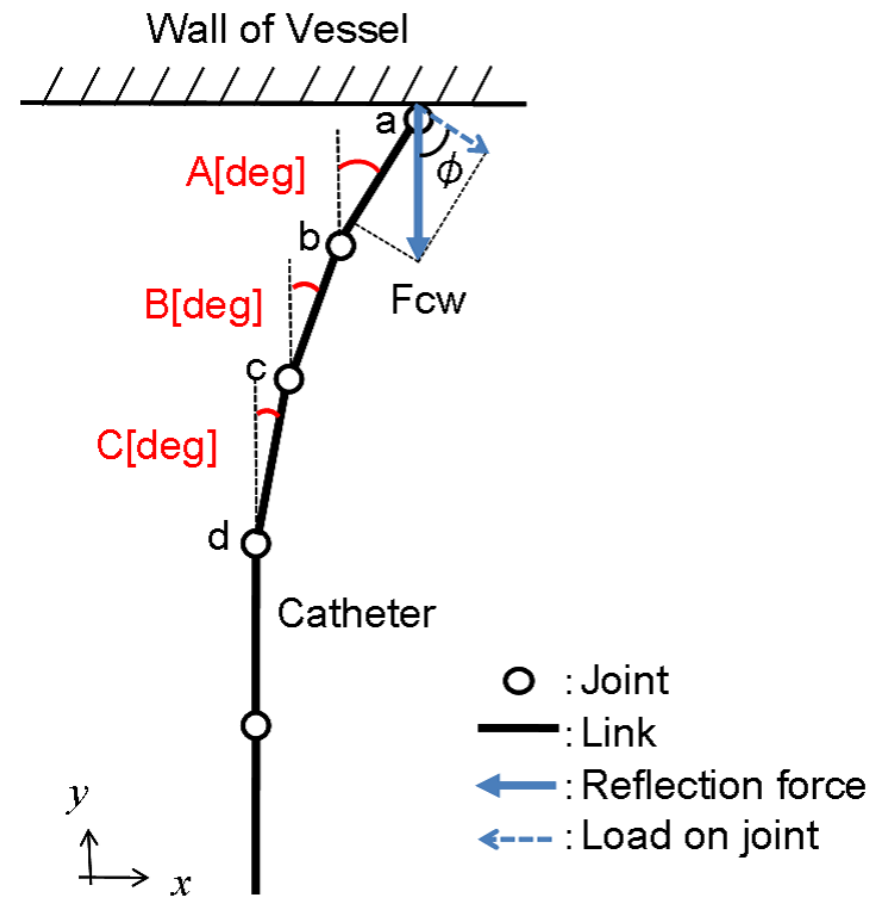

Figure 8: View of the calculation of the catheter inflection.

The program then calculates the inflection angle of the catheter using collision force. Moreover, the reaction force is calculated by acceleration of the catheter. The acceleration is calculated by a second-order differential of displacement of the catheter. The catheter mass is $\mathrm{m}_{c}$; the displacement when there is a collision of the catheter is Xc; the reaction force Fcw is shown in Eq. (2). The mass of the catheter is defined as $10[\mathrm{~g}]$.

As shown in Fig. 8, the inflection angle of the catheter is $\mathrm{K}$ [deg]; the reaction force by collision is Fcw; the catheter length is lc; the longitudinal elastic modulus of the catheter is Ec; and the geometrical moment of inertia is Ic. The angle of the inclination slope of the cantilever equation is shown in Eq. (3). 

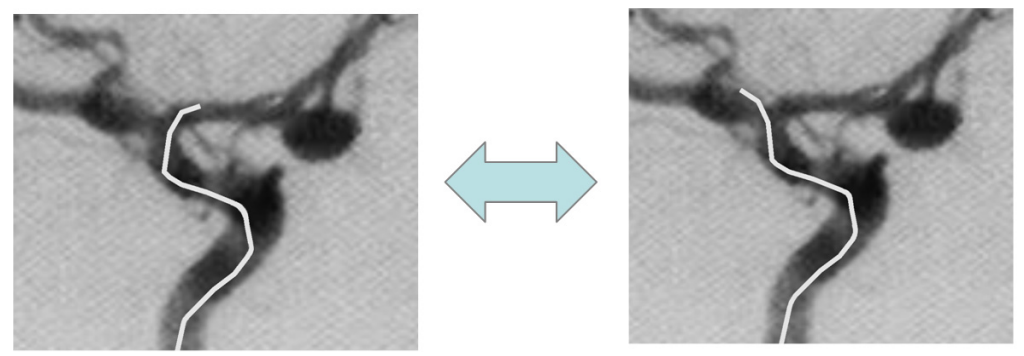

Figure 9: Vascular bifurcation.

$$
\begin{aligned}
& F_{c w}=\left|m_{c} \frac{d^{2} X_{c}}{d t^{2}}\right| \\
& K=\frac{F_{c W} l^{2} \cos \emptyset}{2 E_{c} I_{c}}
\end{aligned}
$$

Link ab of the tip of the catheter revolves A [deg] around joint b. Link bc revolves B [deg] around joint c. Link cd revolves $\mathrm{C}$ [deg] around joint d. After revolution, the detection repeats to the next collision. The guide on the vascular bifurcation is possible, as shown in Fig. 9.

\section{Surgeon's evaluation}

Three surgeons evaluated the training simulator under a variety of conditions. The haptic device was easy to use. The thrust and the rotation of the catheter could be handled instinctively by the surgeon without complicated instructions. The surgeons could use a suitable thrust velocity of the catheter because it could be controlled with the gain. When the catheter came in contact with the vascular wall, the surgeons could evaluate the pressure with the haptic device.

\section{Conclusions}

For this study, a training system was developed for an existing MSS. Moreover, a console box and a simulation program with a connected haptic device were developed. This system can be connected to a PC with a USB port. Its usefulness was assessed by surgeons. In the future, a simulator capable of more detail would enhance the practicality of the system.

\section{Acknowledgements}

The author would like to thank ERtec and the Brain Science and Life Technology Research Foundation. 


\section{References}

[1] Masaru Ide et al.: "Development of a master slave system for interventional radiology", International Journal of Computer Assisted Radiology and Surgery, Volume 3, Supplement 1, pp. 343, 2008

[2] Kostas Vlachos, Evangelos Papadopoulos, Senior Member,Design and Implementation of a Haptic Device for Training in Urological Operations, IEEE TRANSACTIONS ON ROBOTICS AND AUTOMATION, VOL. 19, NO. 5, pp. 801-809

[3] Scott A. Engum, et al.:Intravenous catheter training system: Computerbased education versus traditional learning method The American Journal of surgery 186, pp. 67-74, 2003

[4] F. Arai, R. Fujimura, T. Fukuda, and M. Negoro: "New Catheter Driving Method Using Linear Stepping Mechanism for Intravascular Neurosurgery," Proc. of the IEEE Int. Conf. on Robotics and Automation, pp. 2944-2949, 2002

[5] M.Tanimoto, F.Arai, T. Fukuda: "Force Display Method for Intravascular Neurosurgery," Proc. of the IEEE SMC '99 Conf., Vol. 4, pp. 1032-1037, 1999

[6] Christopher R. Wagner, Robert D.Howe: "Force Feedback Benefit Depends on Experience in Multiple Dgree of Freedom Robotic Surgery Task," IEEE Trans on Robotics, Vol. 23, No. 6, pp. 1235-1240, 2007

[7] Julien Lenoir, Stephane Cotin, at al., Interactive physically-based simulation of catheter and guidewire, Computers \& Graphics 30, pp. 416-422, 2006

[8] Jan Egger, et al., A Fast Vessel Centerline Extraction Algorithm for Catheter Simulation, Twentieth IEEE Int. Symp. on Computer- Based Medical Systems, pp. 177-182

[9] W. Lawton, et al. Tubes in Tubes: Catheter Navigation in Blood Vessels and its Applications, International Journal of Solids and Structures, Vol.37, Issue 22, pp. 3031-3054, 2000

[10] Suraj Bhat, et al., A physically-based model for guidewire simulation on patient-specfic data, International Congress Series, Vol. 1281, pp. 479-484, 2005

[11] Y.Y.Cai, et al., Tactile VR for hand-eye coordination in simulated PTCA, Computers in Biology and Medicine, Vol. 36, pp. 167-180, 2006

[12] DEAGOSTINI FInside Human BodyCUNIT75 